\title{
ABBREVIATIONSAND CONVENTIONS
}

$\begin{array}{ll}\text { ADB } & \text { Asian Development Bank } \\ \text { APEC } & \text { Asia-Pacific Economic Cooperation } \\ \text { CAT } & \text { Convention Against Torture and Other Cruel, Inhuman, or } \\ & \text { Degrading Treatment or Punishment } \\ \text { CEDAW } & \text { Convention on the Elimination of All Forms of Discrimina- } \\ & \text { tion Against Women } \\ \text { CPR } & \text { Center for Prisoners' Rights, Japan } \\ \text { DPJ } & \text { Democratic Party of Japan } \\ \text { ECOSOC } & \text { UN Economic and Social Council } \\ \text { EIA } & \text { Environmental Impact Assessment } \\ \text { EVSL } & \text { Early Voluntary Sector Liberalization } \\ \text { FINRAGE } & \text { Feminist International Network Against Reproductive and } \\ \text { FOM } & \text { Fenetic Engineering } \\ \text { GATS } & \text { General Agreement on Trade in Services } \\ \text { GMO } & \text { Genetically modified organisms } \\ \text { GPPAC } & \text { Global Partnership for the Prevention of Armed Conflict } \\ \text { ICC } & \text { International Criminal Court } \\ \text { ICERD } & \text { Discrimination }\end{array}$


ICFTU International Confederation of Free Trade Unions

IFG International Forum on Globalization

ILO International Labor Organization

IMADR International Movement Against All Forms of Discrimination and Racism

IMF International Monetary Fund

JANIC Japan NGO Center for International Cooperation

JAR Japan Association for Refugees

JCLU Japan Civil Liberties Union

JCP Japan Communist Party

JDA Japan Defense Agency

JICA Japan International Cooperation Agency

JNATIP Japan Network Against Trafficking in Persons

JNICC Japan Network for the International Criminal Court

JNNC Japan NGO Network for CEDAW

JOVC Japan Overseas Volunteer Corps

KSF Kyoto Social Forum

LDP Liberal Democratic Party

LIM Liberalization Impacts Monitor

MAFF Ministry of Agriculture, Forestry and Fisheries

MDG Millennium Development Goals

METI Ministry of Economy, Trade and Industry

MOE Ministry of Environment

MOFA Ministry of Foreign Affairs

NAFTA North American Free Trade Agreement

NAMA Non-Agriculture Market Access

NGO Nongovernmental organization

NPO Nonprofit organization

NPT Nuclear Non-Proliferation Treaty

NWFZ Nuclear-weapon-free zone 


$\begin{array}{ll}\text { ODA } & \text { Overseas Development Assistance } \\ \text { OECD } & \text { Organization for Economic Cooperation and Development } \\ \text { PKO } & \text { Peacekeeping Operations } \\ \text { PPSG } & \text { People's Plan Study Group } \\ \text { PRI } & \text { Penal Reform International } \\ \text { PSI } & \text { Public Services International } \\ \text { RENGO } & \text { Japan Trade Union Confederation } \\ \text { SDF } & \text { Self-Defense Forces } \\ \text { SDP } & \text { Social Democratic Party } \\ \text { TRIPS } & \text { Trade-Related Aspects of Intellectual Property Rights } \\ \text { UNDP } & \text { UN Development Programme } \\ \text { WCAR } & \text { World Conference Against Racism, Racial Discrimination, } \\ & \text { Xenophobia and Related Intolerances } \\ \text { WSF } & \text { World Social Forum } \\ \text { WSFJ } & \text { World Social Forum Japan } \\ \text { WTO } & \text { World Trade Organization } \\ \text { Zenrōkyō } & \text { National Trade Union Council, Japan } \\ \text { Zenrōren } & \text { National Federation of Trade Unions, Japan } \\ \end{array}$

Japanese Names

Japanese names are given in Japanese order: family name first, followed by given name. 

ANOTHER JAPAN ISPOSSIBLE 
